\title{
Immunodiagnostically applicable monoclonal antibodies to the circulating anodic antigen of Schistosoma mansoni bind to small, defined oligosaccharide epitopes
}

Received: 10 February 2003 / Accepted: 28 February 2003/Published online: 15 April 2003

(C) Springer-Verlag 2003

\begin{abstract}
Gut-associated glycoproteins constitute a major group of the circulating excretory antigens produced by human Schistosoma species. The O-glycans of the relatively abundant circulating anodic antigen (CAA) from $S$. mansoni carry long stretches of unique $\rightarrow 6(\mathrm{Glc}$ $\mathrm{A} \beta 1 \rightarrow 3)$ GalNAc $\beta 1 \rightarrow$ repeats. Specific anti-carbohydrate monoclonal antibodies (mAbs) are essential tools for the immunodiagnostic detection of CAA in the serum or urine of Schistosoma-infected subjects. In order to define the epitopes recognised by these anti-CAA mAbs, we screened a series of protein-coupled synthetic di- to pentasaccharide building blocks of the CAA polysaccharide for immunoreactivity, using ELISA and surface plasmon resonance spectroscopy. It was shown that anti-CAA IgM mAbs preferentially recognise $\rightarrow 6(\mathrm{GlcA} \beta 1 \rightarrow 3) \mathrm{Gal}-$ $\mathrm{NAc} \beta 1 \rightarrow$ disaccharide units. Interestingly, no mouse anti-CAA mAbs of the IgG class were found that bind to the synthetic epitopes, although many of the IgG mAbs tested do recognise native CAA in a carbohydratedependent manner. In addition, both IgM and IgG class antibodies could be detected in human infection sera using the synthetic CAA fragments. These synthetic schistosome glycan epitopes and their matching set of specific $\mathrm{mAbs}$ are useful tools that further the development of diagnostic methods and are helpful in defining the immunological responses of the mammalian hosts to schistosome glycoconjugates.
\end{abstract}

H. J. Vermeer · G. J. van Dam · C. H. Hokke $(\square)$

A. M. Deelder

Department of Parasitology,

Center of Infectious Diseases,

Leiden University Medical Center,

PO Box 96002300 RC, Leiden, The Netherlands

E-mail: c.h.hokke@lumc.nl

Tel.: + 31-71-5265065

Fax: + 31-71-5266907

K. M. Halkes · J. P. Kamerling · J. F. G. Vliegenthart

Department of Bio-Organic Chemistry,

Section of Glycoscience and Biocatalysis,

Bijvoet Center, Utrecht University, Padualaan 8,

$3584 \mathrm{CH}$, Utrecht, The Netherlands

\section{Introduction}

Schistosomiasis, which is caused by infection with trematodes of the genus Schistosoma, is one of the most prevalent tropical parasitic diseases. The infection can lead to a debilitating disease with a profound pathology mainly due to granuloma formation caused by schistosome eggs that become lodged in various host organs, particularly the liver. The immunobiology of schistosomiasis comprises complex multi-factorial processes in which many surface antigens and excreted molecules of the parasite play a role. A substantial portion of those molecules that direct the interaction with the host is glycosylated, and it also appears that the major humoral immune response to schistosomes is directed to glycan epitopes on glycoconjugate antigens of different stages of the life-cycle (Cummings and Nyame 1999; Hokke and Deelder 2001). Abundantly expressed antigens include the gut-associated excretory antigens which are released into the circulation of the host at regular time intervals from the gut of adult schistosomes (Nash et al. 1977; Carlier et al. 1980; Deelder et al. 1980). Quantitative immunodiagnostic techniques based on the detection of these and other circulating antigens in the urine or serum of infected subjects are increasingly recognised as alternatives to classical methods for the diagnosis of schistosomiasis (De Jonge et al. 1989; Nourel Din et al. 1994; Van Lieshout et al. 2000). Two antigens which have been extensively studied, primarily as immunodiagnostic targets, are the circulating anodic antigen (CAA), a proteoglycan with a strong negative charge at neutral $\mathrm{pH}$, and the circulating cathodic antigen (CCA), a glycoprotein that is neutral or slightly positively charged at neutral $\mathrm{pH}$ (Berggren and Weller 1967; Nash et al. 1977; Carlier et al. 1980; Deelder et al. 1980). Both antigens are demonstrable in the serum and urine of hosts infected with Schistosoma mansoni (Nash 1974; Deelder et al. 1978). The roles of CCA and CAA in the biology of schistosomes are not clear. It has been suggested that CAA may serve as a lining to protect the 
331

schistosome gut against harmful host components. On the other hand, it may also have immunomodulatory functions, e.g. by interaction with the complement sssterm of the host (Van Dam et al. 1993). About 30\% by weight of CAA from S. manson is carbohydrate, and the major immunogenic character of CAA is carried by an O-linked polysaccharide composed of multiple repeats of the $\rightarrow 6(\mathrm{GlcA} \beta 1 \rightarrow 3)$ GalNAc $\beta 1 \rightarrow$ disaccharide element (Fig. 1) (Bergwerff et al. 1994).

A panel of anti-CAA mAs has been produced over the years from fused spleen cells of mice infected with

Fig. 1 Polysaccharide chain of CAA of Schistosome mansoni, and conjugated synthetic oligosaccharide fragments. Structure 1: schematic representation of the CAA polysaccharide; CAA contains at least 30 repeating units. Structures 2-5 Oligosaccharide fragments coupled to BSA. Structure 2 DiCAA, 3 TriCAA, 4 TetCAA, and 5 PenCAA
Schistosome or immunised with schistosome-derived antigen preparations. Most of these dAbs have been shown to recognise CAA in a carbohydrate-dependent way (Deelder et al. 1996). Immunodiagnostic detection of CAA results in a nearly $100 \%$ specificity (Deelder and Kornelis 1981; Deelder et al. 1989), and it was hypothesised that this is due to the unique repetitive $\rightarrow 6(\mathrm{GlcA} \beta 1 \rightarrow 3) \mathrm{GalNAc} \beta 1 \rightarrow$ units of CAA. To structurally define the carbohydrate epitomes involved in immunorecognition by anti-CAA mAds, di- to pentasaccharide fragments of the major CAA polysaccharide chain were synthesised and subsequently conjugated to bovine serum albumin (BSA) to yield a series of defined neoglycoconjugates (Fig. 1) (Hakes et al. 1998; Vermeer et al. 2000). In the current study, interactions of antiCAA mAds with these glycoconjugates were evaluated by ELISA and by surface plasmon resonance (SPR) spectroscopy (BIAcore).

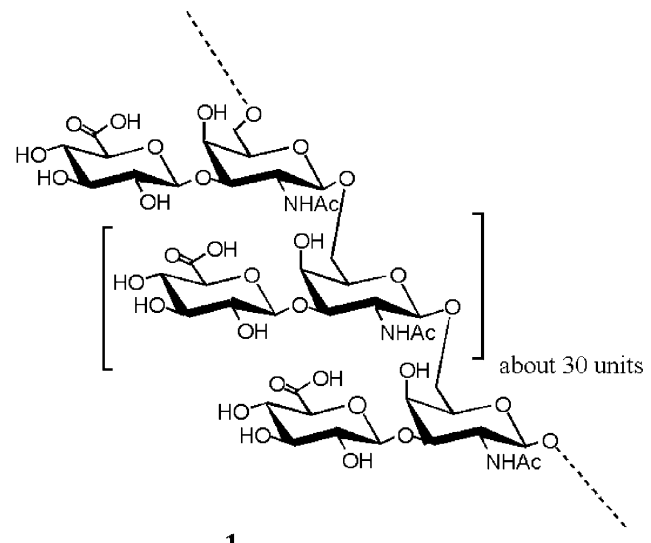

1

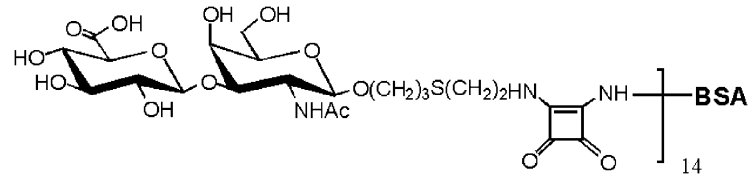

2

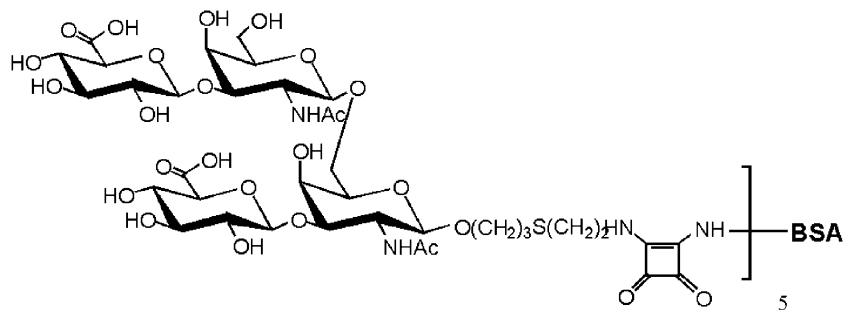

4

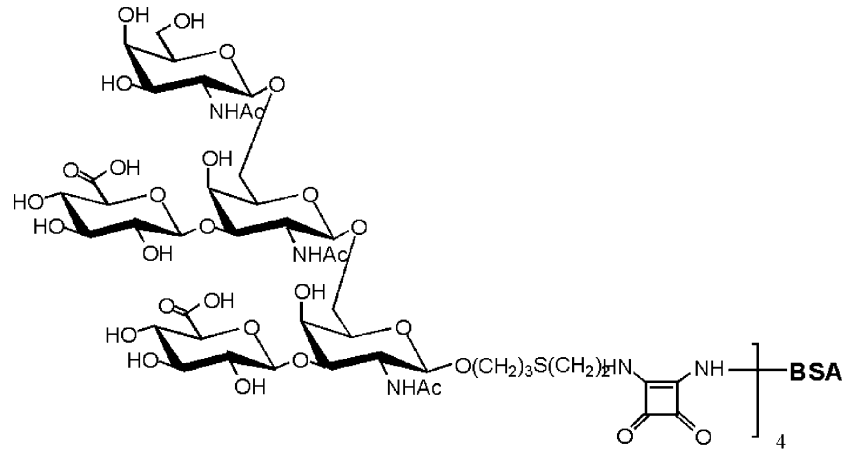

5 


\section{Materials and methods}

Preparation of the neoglycoconjugates

The synthesis of spacer-containing di- (DiCAA), tri- (TriCAA), tetra- (TetCAA) and pentasaccharide (PenCAA) fragments of CAA and the subsequent conversion to BSA conjugates have been reported previously (Halkes et al. 1998; Vermeer et al. 2000).

Production of monoclonal antibodies

MAbs directed against CAA were produced from hybridomas derived from the spleen cells of schistosome-infected mice or mice immunised with antigen preparations of S. mansoni, Schistosoma haematobium, or Schistosoma japonicum as described by Deelder et al. (1996). A panel of $24 \mathrm{mAbs}$, all directed against CAA as determined by immunoelectrophoresis and immunolocalisation (Deelder et al. 1996), was used for this study. Culture supernatants containing the antibody were used without further purification to study interactions with the synthetic epitopes, unless otherwise stated. Concentrations of mAbs used for the SPR measurements were determined by ELISA.

\section{ELISA screening}

Indirect ELISAs were carried out to determine the binding of anti$\mathrm{CAA} \mathrm{mAbs}$ to the neoglycoconjugates. Inhibition ELISAs were performed to study the competitive effect of trichloroacetic acidtreated adult worm antigen (AWA-TCA, which contains native CAA) on three selected mAbs. In general, unconjugated BSA was taken as a negative control, incubations were kept for $15 \mathrm{~min}$ at $37^{\circ} \mathrm{C}$, while plates (Maxisorp, Nunc, Roskilde, Denmark) were shaken (Mushens and Scott 1990), and absorbance readings were made at $630 \mathrm{~nm}\left(A_{630}\right)$ within $5 \mathrm{~min}$ of the addition of the substrate, with occasional shaking of the plates to ensure linear peroxidase reactivity.

\section{Indirect ELISA}

Neoglycoconjugates, AWA-TCA, and control BSA were coated to the plate in phosphate buffered saline (PBS) at $5 \mu \mathrm{g} / \mathrm{ml}$. Wells were washed with diluted PBS (1:20) and incubated with hybridoma supernatants in $0.3 \%$ Tween-20 in PBS (PT). After washing, the plates were incubated with peroxidase-conjugated rabbit antimouse $\mathrm{F}\left(\mathrm{ab}^{\prime}\right)_{2}$ fragments (Dakopatts, Denmark) $1 / 1000$ in PT. After a thorough final washing, the peroxidase substrate solution [0.42 mM 3,3',5,5'-tetramethylbenzidine (TMB; Polysciences, Warrington, USA), $1.4 \mathrm{mM} \mathrm{H}_{2} \mathrm{O}_{2}$ in $0.1 \mathrm{M}$ sodium acetate buffer pH 5.5 (Hancock and Tsang 1986)] was added, and $A_{630}$ measured. The TMB and $\mathrm{H}_{2} \mathrm{O}_{2}$ concentrations used were shown to be present in excess amounts (Bos et al. 1981). At least three $A_{630}$ values were determined within $5 \mathrm{~min}$, and transformed into plots of $\delta\left(A_{630}\right)$ values versus time.

\section{Inhibition ELISA}

Neoglycoconjugates were coated to the plate as described for the indirect ELISA. Before addition to the wells, $\mathrm{mAbs}$ were preincubated with various concentrations of AWA-TCA for $20 \mathrm{~min}$ at $37^{\circ} \mathrm{C}$. The inhibition ELISA was further carried out as described for the indirect ELISA. Results were expressed as $A_{630}$ values, and the percentage inhibition was plotted against the inhibitor concentration.

Human serum samples

Serum samples were obtained from Dutch and Surinamese individuals who underwent immunodiagnosis for schistosomiasis at the clinical laboratory of the Leiden University Medical Center. Two routinely used immunoassays (IFA and ELISA, Nash 1974, Deelder et al. 1980) were performed to diagnose the infection. No further details concerning infection intensity were available. Sera from healthy Dutch donors were used as negative controls.

\section{Surface plasmon resonance studies}

SPR studies were carried out using a BIAcore 3000 instrument (BIAcore, Uppsala, Sweden). The Sensor Chip CM5 and Amine Coupling Kit were also obtained from BIAcore. The neoglycoconjugates were immobilised at a flow rate of $5 \mu \mathrm{l} / \mathrm{min}$ in $10 \mathrm{mM}$ sodium acetate $(\mathrm{pH} 4.0)$ onto a carboxylmethylated dextran CM5 sensor chip by covalent amine coupling until an increase of approximately 3,500-4,500 response units (RU) was observed using described procedures (Van Remoortere et al. 2000, 2001a). On each CM5 sensor chip there was also a control BSA channel prepared to correct for the buffer-related RU change on injection, matrix effects and non-specific interactions (Myszka 1999). For binding experiments, $5 \mu$ l of undiluted hybridoma supernatant were injected using HEPES-buffered saline (HBS) as an eluent at $25^{\circ} \mathrm{C}$ and at flow rates of $5 \mu \mathrm{l} / \mathrm{min}$. Surfaces were regenerated with a $240 \mathrm{~s}$ pulse of $20 \mathrm{mM} \mathrm{HCl}$. Several mAbs were subsequently injected at different concentrations at a flow rate of $10 \mu \mathrm{l} / \mathrm{min}$ to study the concentration dependence of the binding.

\section{Results}

\section{Screening of anti-CAA mAbs by ELISA}

A panel of $24 \mathrm{mAbs}$ that bind to CAA was screened for immunoreactivity with the BSA-conjugated DiCAA, TriCAA, TetCAA and PenCAA, and with AWA-TCA as a positive control. The level of CAA in AWA-TCA is estimated to be in the order of $2.5 \% \mathrm{w} / \mathrm{w}$ (Bergwerff et al. 1994). Binding of the mAbs to the neoglycoconjugates was first tested by indirect ELISA using undiluted hybridoma culture supernatants. After calculation of the slopes of plots of $\delta\left(A_{630}\right)$ values versus time for samples (S) and native BSA (R), results were expressed as $\mathrm{S}$-minus R-values (Table 1).

A number of anti-CAA mAbs specifically recognise the synthetic carbohydrate epitopes. The mAbs binding to the neoglycoconjugates are restricted essentially to the IgM isotype. In most cases, DiCAA, TetCAA, and PenCAA were bound moderately to strongly by $\operatorname{IgM}$ antibodies, whereas TriCAA gave negative or borderline results. In general, insignificant binding to the neoglycoconjugates was observed for the $\mathrm{IgG}$ isotypes, except 120-1B6-A and 145-3C6-A.

\section{Inhibition ELISA}

Inhibition ELISA was carried out in order to determine the inhibitory effect of AWA-TCA on the binding of $\mathrm{mAbs}$ to the synthetic carbohydrate epitopes. The anti-CAA mAbs 27-2E2-A (IgM), 25-9B10-A (IgM) and 147-1B4-A (IgG) were studied in the inhibition assay (Table 2). The concentration of AWA-TCA necessary for the $50 \%$ inhibition $\left(I_{50}\right)$ of binding of 27-2E2-A and 25-9B10-A to DiCAA, TetCAA, and PenCAA was in the range of $0.3-3 \mu \mathrm{g} / \mathrm{ml}$, which corresponds to 
Table 1 ELISA screening of anti-CAA mAbsfor binding with neoglycoconjugates. Values are expressed as the slope of $\delta\left(A_{630}\right)$ plots versus time $(0-5 \mathrm{~min})$, corrected for native BSA as background. AWA-TCA denotes the trichloroacetic acid-soluble fraction of total adult worm antigen

\begin{tabular}{|c|c|c|c|c|c|c|}
\hline \multirow[t]{2}{*}{$\mathrm{mAb}$} & \multirow[t]{2}{*}{ Isotype } & \multicolumn{4}{|c|}{ BSAconjugate } & \multirow{2}{*}{$\begin{array}{l}\text { AWA- } \\
\text { TCA }\end{array}$} \\
\hline & & DiCAA & TriCAA & TetCAA & PenCAA & \\
\hline $5-25-\mathrm{B}$ & M & 9 & 2 & 112 & 118 & 47 \\
\hline $25-2 \mathrm{~B} 6-\mathrm{A}$ & M & 33 & 0 & 37 & 15 & 79 \\
\hline 25-7C11-A & M & 57 & 0 & 57 & 26 & 78 \\
\hline 25-9B10-A & $\mathrm{M}$ & 66 & 5 & 77 & 36 & 82 \\
\hline 27-2E2-A & M & 49 & 4 & 55 & 14 & 77 \\
\hline 25-3D10-A & M & 98 & 2 & 109 & 60 & 154 \\
\hline 120-1C4-A & M & 5 & 1 & 41 & 54 & 197 \\
\hline 51-4B3-D & G3 & 1 & 1 & 0 & 0 & 54 \\
\hline 51-4G5-A & G3 & 1 & 2 & 0 & 0 & 47 \\
\hline $54-5 \mathrm{C} 5-\mathrm{B}$ & G3 & 0 & 0 & 0 & 0 & 51 \\
\hline 54-5G10-A & G1 & 0 & 1 & 5 & 3 & 99 \\
\hline 114-4E10-A & G1 & 0 & 1 & 7 & 3 & 98 \\
\hline $120-1 \mathrm{C} 2-\mathrm{C}$ & G1 & 3 & 2 & 3 & 2 & 94 \\
\hline $120-1 \mathrm{C} 11-\mathrm{C}$ & G & 0 & 0 & 5 & 1 & 97 \\
\hline 141-2A9-A & G1 & 0 & 0 & 0 & 0 & 94 \\
\hline 141-2F8-A & G & 0 & 0 & 5 & 5 & 99 \\
\hline 145-2G1 & G1 & 1 & 1 & 1 & 3 & 98 \\
\hline 147-1B1-B & G1 & 0 & 0 & 3 & 2 & 103 \\
\hline 147-1B4-A & G1 & 1 & 3 & 12 & 7 & 201 \\
\hline 120-1B6-A & $\mathrm{G}$ & 1 & 1 & 14 & 55 & 202 \\
\hline 120-1B10-A & G1 & 0 & 0 & 0 & 0 & 202 \\
\hline 145-3C6-A & G & 2 & 2 & 23 & 13 & 199 \\
\hline 147-4E5-A & G & 3 & 1 & 2 & 3 & 213 \\
\hline 141-5C9-A & G & 2 & 2 & 10 & 9 & 213 \\
\hline
\end{tabular}

Table 2 Inhibition of anti-CAA mAb binding to coated neoglycoconjugates by AWA-TCA. $I_{50}$ is the estimated concentration of AWA-TCA at which the mAb showed a $50 \%$ decrease in binding. The concentration of the mAbs is normalized to $1 \mu \mathrm{g} / \mathrm{ml}$. n.d. stands for not detectable, and denotes that no affinity of the $\mathrm{mAb}$ for the coated antigen fragments was observed

\begin{tabular}{|c|c|c|c|c|c|}
\hline \multirow[t]{2}{*}{$\mathrm{mAb}$} & \multirow[t]{2}{*}{ Isotype } & \multicolumn{4}{|c|}{$I_{50}(\mu \mathrm{g} / \mathrm{ml})$} \\
\hline & & DiCAA & TriCAA & TetCAA & PenCAA \\
\hline $27-2 \mathrm{E} 2-\mathrm{A}$ & $\operatorname{IgM}$ & 2.5 & $\begin{array}{l}\text { not } \\
\text { detectable }\end{array}$ & 3 & 2.5 \\
\hline 25-9B10-A & $\operatorname{IgM}$ & 0.9 & n.d. & 1.9 & 0.3 \\
\hline 147-1B4-A & IgG1 & n.d. & n.d. & n.d. & n.d. \\
\hline
\end{tabular}

7.5-75 ng/ml of CAA. No significant data were obtained for IgG mAb 147-1B4-A, which bound only relatively weakly to Di-, Tri-, Tetra- or PenCAA.

Evaluation of $\mathrm{mAb}$ - glycoconjugate interaction by SPR analysis

For a more extensive characterisation of the interactions between mAbs and CAA glycan fragments, and for validation of the ELISA-screening, the binding of the 24 anti-CAA mAbs with the neoglycoconjugates immobilised on BIAcore sensor surfaces was studied. No non-specific binding of the mAbs to the BSA control reference surface was observed. Regarding the specificity of the anti-CAA mAbs, the BIAcore data supported the outcome of the indirect ELISAs. No significant binding could be detected in the case of the TriCAA-conjugate, and none of the tested IgG subclass $\mathrm{mAbs}$ bound to any of the tested glycoconjugates (data not shown).

The concentration dependent characteristics of the $\mathrm{mAb}$-glycoconjugate interactions were further studied by the injection of the antibodies to the sensor surfaces at five different concentrations. Contact times were $420 \mathrm{~s}$ for the association phase, and $640 \mathrm{~s}$ for the dissociation phase, at a constant flow rate of $10 \mu \mathrm{l} / \mathrm{min}$. In Fig. 2, overlay plots are presented to illustrate the concentration-dependent binding characteristics of several mAbs. Injection of the mAbs on to sensor surface channels carrying DiCAA and TetCAA showed comparable results, whereas the PenCAA surface gave somewhat lower binding responses. Kinetic analysis to calculate the absolute affinities of the different antibody-carbohydrate interactions did not give reliable results (data not shown). The IgM antibodies and the neoglycoconjugates are multivalent analytes whereas monovalent analytes would be required for accurate kinetic analysis of affinities by SPR. Depending on the antibody-antigen combination studied, the apparent kinetic rate constants were in the $10^{5}-10^{8} \mathrm{M}^{-1} \mathrm{~s}^{-1}$ range for $k_{\mathrm{a}}$ and $10^{-2}$ $10^{-4} \mathrm{~s}^{-1}$ range for $k_{\mathrm{d}}$, giving rise to $K_{\mathrm{A}}$ values of $10^{6}-10^{10} \mathrm{M}^{-1}$, which is within the normal range for antigen-antibody interactions.

Immunoreactivity of infection sera with the glycoconjugates

To test the potential of the synthetic oligosaccharides for the detection of serum antibodies, sera obtained from schistosome-infected individuals $(n=67)$ and healthy controls $(n=5)$ were subjected to BIAcore determination of specific IgM and IgG levels according to the previously reported methods (Van Remoortere et al. 2000, 2001a). The infection sera were studied by IFA and ELISA to determine the presence of IgM against $S$. mansoni male adult worms and of $\mathrm{IgG}$ against $S$. mansoni soluble egg antigens, respectively, and subsequently categorised into four groups according to the antibody titers (Nash 1974; Deelder et al. 1980). For each of these groups, the antibody response against TriCAA, TetCAA and PenCAA was evaluated (Table 3). In Fig. 3, an example of an overlay plot of BIAcore sensorgrams is given that illustrates the binding of serum antibodies to the synthetic compounds, corrected for BSA background binding, and including the $\operatorname{IgM} / \operatorname{IgG}$ determination. Apparently, in contrast to the observations for the mouse mAbs, in infection serum the IgG responses are equally significant as those of IgM. Similarly, it appears that serum responses are in the same range for all conjugates tested, whereas no significant binding could be detected with TriCAA in case of the mAbs. 

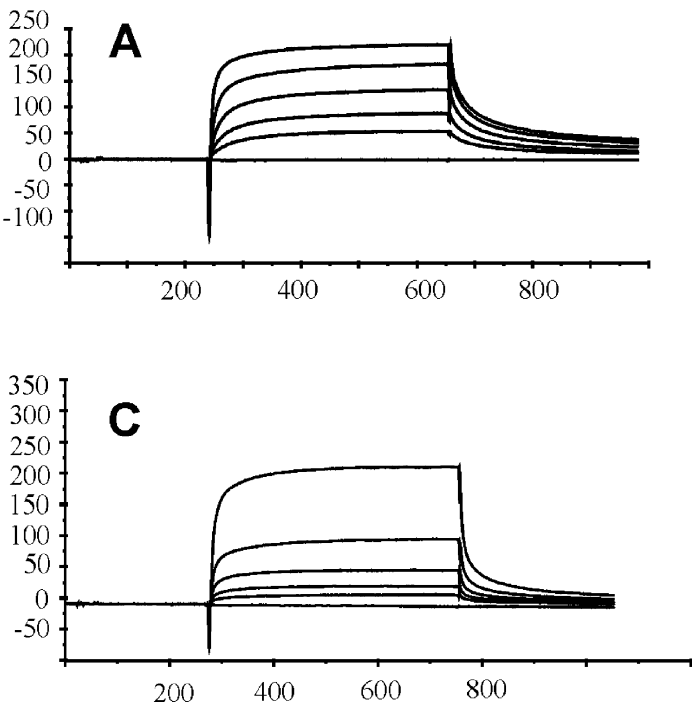

Fig. 2a-d Surface plasmon resonance (BIAcore) response plots at different concentrations of anti-CAA mAbs binding to immobilised DiCAA, TetCAA or PenCAA. a Overlay plot of interaction of $\mathrm{mAb} 27-2 \mathrm{E} 2-\mathrm{A}$ with TetCAA; from top to bottom curve, the mAb concentration was: $0.70,0.35,0.17,0.09$ and $0.04 \mathrm{nM}$. b Interaction of $\mathrm{mAb} 5-25-\mathrm{B}$ with PenCAA; mAb concentration: $0.64,0.32,0.16$, 0.08 and $0.04 \mathrm{nM}$. c Interaction of 25-7C11-A with DiCAA; mAb concentration: $0.56,0.28,0.14,0.07$ and $0.03 \mathrm{nM}$. d Interaction of 25-9B10-A with TetCAA; mAb concentration: 0.36, 0.18, 0.09, 0.05 and $0.02 \mathrm{nM}$

Table 3 Numbers of sera (out of $n$ ) positive for total serum, IgM or IgG response to TriCAA, TetCAA or PenCAA, determined by SPR. Category determined by IFA and ELISA analysis, grouped according to antibody titers:,$+ 8-32 ;++, 64-128 ;+++, 256-$ $512 ;++++, 1024-2048$

\begin{tabular}{llllll}
\hline Category & Conjugate & $n$ sera & Total serum & IgG & IgM \\
\hline+ & TriCAA & 18 & 8 & 8 & 12 \\
& TetCAA & 18 & 5 & 3 & 13 \\
++ & PenCAA & 18 & 3 & 3 & 3 \\
& TriCAA & 21 & 16 & 12 & 13 \\
& TetCAA & 21 & 15 & 9 & 12 \\
+++ & PenCAA & 21 & 10 & 10 & 8 \\
& TriCAA & 21 & 18 & 17 & 14 \\
++++ & TetCAA & 21 & 19 & 15 & 13 \\
& PenCAA & 21 & 15 & 12 & 11 \\
& TriCAA & 7 & 7 & 6 & 7 \\
& TetCAA & 7 & 7 & 6 & 7 \\
& PenCAA & 7 & 7 & 5 & 6 \\
\hline
\end{tabular}

\section{Discussion}

The structural identification of the immunoreactive part of schistosome glycans is required for understanding the immunological responses of the host against glycoconjugate antigens of the parasite. Moreover, specific antibodies to such antigens can serve as valuable diagnostic and analytical tools. In the case of schistosome infections, the main alternative diagnostic techniques to faecal or urinary egg counts are based on the quantita-
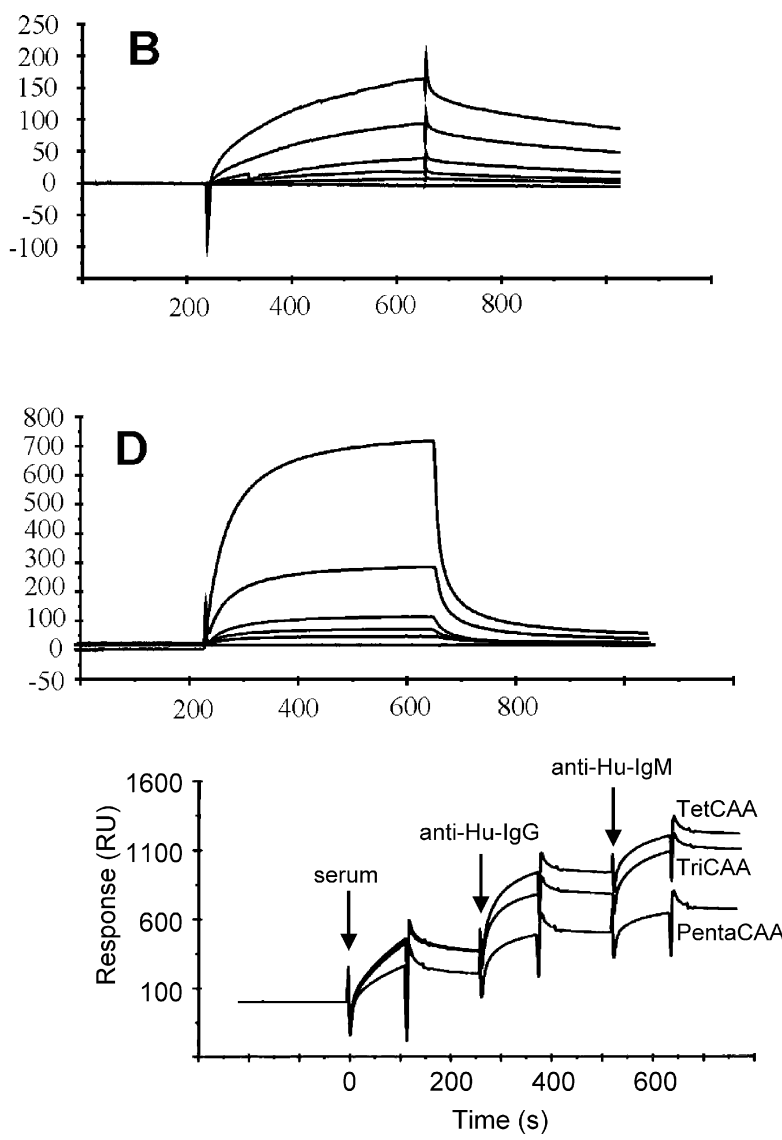

Fig. 3 Overlay plot of SPR sensorgrams illustrating the binding of antibodies from human schistosomiasis infection serum to synthetic CAA fragments. The arrows indicate the time point of injection of the serum, goat anti-human IgG and goat anti-human IgM, respectively

tive determination of circulating antigens using antigen specific mAbs. The immunodiagnostic detection of schistosome antigens, including CAA in serum, has been developed to a routinely applicable technique and is now increasingly used for general applications in the diagnosis of schistosome infections, as well as for basic academic studies (Van Lieshout et al. 2000).

The major purpose of the current study was to evaluate the determinants on the main CAA polysaccharide that are recognised by anti-CAA mAbs. To this end, small synthetic oligosaccharides were coupled to a carrier protein to create the multivalent epitope presentation that is necessary for the affinity enhancement generally observed in interactions between carbohydrates and proteins, including antibodies. The free accessibility of the antibodies to the oligosaccharides was accomplished by the spatial separation of the oligosaccharide from the carrier protein using a nine-atom spacer moiety (Fig. 1).

The ELISA and SPR data show that several antiCAA mAbs of the IgM class recognise the disaccharide unit GlcA $\beta 1 \rightarrow 3$ GalNAc (DiCAA) that forms the repeating element of the CAA polysaccharide. In this context, it was of interest to determine whether the anti- 
CAA mAbs had a higher affinity for a larger epitope consisting of two repeating units, i.e. TetCAA. Unfortunately, the high avidity of the IgM analytes implies that the requirements for the pseudo-first order kinetics necessary to derive accurate SPR kinetic data can not be fulfilled (MacKenzie et al. 1996). In fact, kinetic analysis using multivalent analytes can cause large discrepancies with thermodynamic binding constants obtained from homogeneous solution methods (Nieba et al. 1996), whereas the rebinding effects of the eluting multivalent IgM antibodies can further obscure calculated binding constants in the dissociation phase. Therefore, quantitative data to evaluate the difference in affinity for binding to DiCAA or TetCAA cannot be achieved at present, but qualitative examination and comparison of the responses for binding to DiCAA and TetCAA suggests comparable affinities of $\mathrm{mAbs}$ for the two different epitopes. Clearly, these results indicate that the antibodies directed against CAA require only one repeating unit of the polysaccharide for efficient occupation of their binding site. No significant immunoreactivity of any of the mAbs tested was found toward the TriCAA epitope. Apparently, the presence of a terminal nonsubstituted GalNAc residue in the oligosaccharide hinders binding of the $\mathrm{mAb}$ to the underlying disaccharide unit. This effect is also illustrated by the lower binding levels observed for PenCAA compared to TetCAA.

It is surprising that the majority of the anti-CAA mAbs of the IgG isotype did not bind to any of the epitopes tested. Of the anti-CAA mAbs generated in mice, $60 \%$ were of the IgG1 isotype and anti-CAA IgG mAbs effectively bind native CAA in immunoassays (Deelder et al. 1996 and Table 2). The carbohydrate part of CAA is thought to be almost solely responsible for the strong immunogenicity of this parasite antigen since TCA treatment of antigen preparations does not destroy the immunoreactivity with anti-CAA mAbs. It was previously suggested that the unique $\rightarrow 6(\mathrm{GlcA} \beta 1 \rightarrow 3)$ GalNAc $\beta 1 \rightarrow$ polysaccharide sequence in the $\mathrm{O}$-glycans of CAA is the main antigenic element (Bergwerff et al. 1994). It is not clear why none of the IgG type mAbs tested here bind to the synthetic glycan epitopes. A potential explanation could be that the $\operatorname{IgG} \mathrm{mAbs}$ preferentially bind to other CAA-related glycan epitopes. It has been reported that $5 \%$ or less of the CAA glycans do not contain the $\rightarrow 6(\mathrm{GlcA} \beta 1 \rightarrow 3)$ GalNAc $\beta 1 \rightarrow$ polysaccharide sequence. These glycans may contain the Lewis $\mathrm{X}$ repeats that are characteristic for CCA and account for the cross-reactivity of anti-CCA mAbs with CAA (Bergwerff et al. 1994). An additional hypothesis may be that IgG mAbs preferentially bind to $\rightarrow 6(\mathrm{GlcA} \beta 1 \rightarrow 3) \mathrm{Gal}-$ $\mathrm{NAc} \beta 1 \rightarrow$ multimers longer than those tested. Several IgG mAbs (147-1B4-A, 120-1B6-A, 145-3C6-A and 1415C9-A) bind moderately well to the TetCAA and PenCAA conjugates, whereas none bind to DiCAA and TriCAA (Table 2). This observation indeed suggests a tendency for anti-CAA IgG mAbs to bind more effectively to longer CAA oligosaccharides, which may partly be due to a higher ordered structure of the longer polymer (Bergwerff et al. 1994; Vermeer et al. 2000 ).

We observed that from a panel of sera obtained from schistosome-infected humans, the majority contained IgM as well as IgG antibodies to TriCAA and in particular TetCAA and PenCAA (Table 3). This is surprising in view of the fact that almost no IgG class $\mathrm{mAbs}$ bind to the synthetic CAA fragments. Another possible explanation for this apparently contrasting observation may be that the mouse mAbs that were first selected against isolated, intact CAA did not include any IgG reactive to small oligosaccharides in spite of the initial occurrence of these antibodies before selection. To explore the potential of anti-carbohydrate antibodies as markers of infection, studies have been initiated to determine antibody reactivities towards the CAA neoglycoconjugates in the serum of experimentally infected animals. So far it has been shown that chimpanzees infected with $S$. mansoni produce both $\operatorname{IgM}$ and IgG to TetCAA, in particular early during infection (Van Remoortere et al. 2001b). Other synthetic immunogenic schistosome oligosaccharides including Lewis $\mathrm{X}$, GalNAc $\beta 1 \rightarrow 4 \mathrm{GlcNAc}$ and Gal$\mathrm{NAc} \beta 1 \rightarrow 4(\mathrm{Fuc} \alpha 1 \rightarrow 2 \mathrm{Fuc} \alpha 1 \rightarrow 3)$ GlcNAc were successfully applied to monitor antibody responses in S. mansoni, S. japonicum, or S. haematobium-infected patients (Van Remoortere et al. 2001a). In addition, these oligosaccharides allowed the characterisation of schistosome-specific mAbs similar to the current study, and it appeared that generally both $\operatorname{IgM}$ and $\operatorname{IgG}$ type mAbs specific for a particular oligosaccharide epitope occurred (Van Remoortere et al. 2000).

ELISA and SPR techniques provide simple and rapid mutually corroborating means for studying the binding of mAbs to synthetic glycan epitopes, and most probably antigens in general. In the case of scarcely available and expensive synthetic glycoconjugates like the CAA fragments, SPR is proving to be particularly useful. In conclusion, the synthetic glycoconjugates that represent parts of the CAA-derived polysaccharide, and the identification described here of mAbs immunoreactive with these small glycan epitopes, are important tools that will facilitate further research on schistosome glycan antigens as well as the humoral immune responses in the host.

Acknowledgements The authors wish to thank Dr. S. Haseley for critical reading of the manuscript, and Ms. A. van Remoortere for helpful discussions. The technical assistance of Ms. D. Kornelis, Mr. R.J.M. van Zeyl, and Ms. S. Ferreira is highly appreciated.

\section{References}

Berggren WL, Weller TH (1967) Immunoelectrophoretic demonstration of specific circulating antigen in animals infected with Schistosoma mansoni. Am J Trop Med Hyg 16:606-612

Bergwerff AA, van Dam GJ, Rotmans JP, Deelder AM, Kamerling JP, Vliegenthart JFG (1994) The immunologically reactive part of immunopurified circulating anodic antigen from Schistosoma 
mansoni is a threonine-linked polysaccharide consisting of $\rightarrow 6)-[\beta$-D-GlcpA- $(1 \rightarrow 3)]-\beta$-D-Gal $p N A c-(1 \rightarrow \quad$ repeating units. J Biol Chem 269:31510-31517

Bos E, van der Doelen A, van Rooy N, Schuurs A (1981) 3,3',5,5'tetramethylbenzidine as an Ames test negative chromogen for horseradish peroxidase in enzyme immunoassay. J Immunoassays 2:187-196

Carlier Y, Bout D, Strecker G, Debray H, Capron A. (1980) Purification, immunochemical, and biological characterisation of the Schistosoma circulating M antigen. J Immunol 124:24422450

Cummings RD, Nyame AK (1999) Schistosome glycoconjugates. Biochim Biophys Acta 1455:363-374

De Jonge N, de Caluwé P, Hilberath GW, Krijger FW, Polderman AM, Deelder AM (1989) Circulating anodic antigen levels in serum before and after chemotherapy with praziquantel in Schistosoma mansoni. Trans R Soc Trop Med Hyg 83:368-372

Deelder AM, Kornelis D (1981) Immunodiagnosis of recently acquired Schistosoma mansoni infection. A comparison of various immunological techniques. Trop Geogr Med 33:36-41

Deelder AM, van Dalen DP, van Egmond JG (1978) Schistosoma mansoni: microfluorometric determination of circulating anodic antigen and antigen-antibody complexes in infected hamster serum. Exp Parasitol 44:216-224

Deelder AM, Kornelis D, van Marck EAE, Eveleigh PC, van Egmond JG (1980) Schistosoma mansoni: characterisation of two circulating polysaccharide antigens and the immunological response to these antigens in mouse, hamster, and human infections. Exp Parasitol 50:16-32

Deelder AM, de Jonge N, Boerman OC, Fillié YE, Hilberath GW, Rotmans JP, Gerritse MJ, Schut DWOA (1989) Sensitive determination of circulating anodic antigen in Schistosoma mansoni infected individuals by an enzyme-linked immunosorbent assay using monoclonal antibodies. Am J Trop Med Hyg 40:268-272

Deelder AM, van Dam GJ, Kornelis D, Fillié YE, van Zeyl RJM (1996) Schistosoma: analysis of monoclonal antibodies reactive with the circulating antigens CAA and CCA. Parasitology 112:21-35

Halkes KM, Vermeer HJ, Slaghek TM, van Hooft PAV, Loof A, Kamerling JP, Vliegenthart JFG (1998) Preparation of spacercontaining di-, tri-, and tetrasaccharide fragments of the circulating anodic antigen of Schistosoma mansoni for diagnostic purposes. Carbohydr Res 309:175-188

Hancock K, Tsang VCW (1986) Development and optimization of the FAST-ELISA for detecting antibodies to Schistosoma mansoni. J Immunol Methods 92:167-176

Hokke CH, Deelder AM (2001) Schistosome glycoconjugates in host-parasite interplay. Glycoconj J 18:573-587

MacKenzie CR, Hirama T, Deng S-J, Bundle DR, Narang SA, Young NM (1996) Analysis by surface plasmon resonance of the influence of valence on the ligand binding affinity and kinetics of an anti-carbohydrate antibody. J Biol Chem $271: 1527-1533$
Mushens RE, Scott ML (1990) A fast and efficient method for quantification of monoclonal antibodies in an ELISA using a novel incubation system. J Immunol Methods 131:83-89

Myszka DG (1999) Improving biosensor analysis. J Mol Recognit $12: 1-6$

Nash TE (1974) Localization of the circulating antigen within the gut of Schistosoma mansoni. Am J Trop Med Hyg 23:1085-1087

Nash TE, Nasir-ud-din, Jeanloz RW (1977) Further purification and characterisation of a circulating antigen in schistosomiasis. J Immunol 119:1627-1633

Nieba L, Krebber A, Plückthun A (1996) Competition BIAcore for measuring true affinities: large differences from values determined from binding kinetics. Anal Biochem 234:155-165

Nourel Din MS, Nibbeling R, Rotmans JP, Polderman AM, Krijger FW, Deelder AM (1994) Quantitative determination of circulating soluble egg antigen in urine and serum of Schistosoma mansoni-infected individuals using a combined two-site enzyme-linked immunosorbent assay. Am J Trop Med Hyg 50:585-94

Van Dam GJ, Seino J, Rotmans JP, Daha MR, Deelder AM (1993) Schistosoma mansoni circulating anodic antigen but not circulating cathodic antigen interacts with complement component C1q. Eur J Immunol 23:2807-2812

Van Lieshout L, Polderman AM, Deelder AM (2000) Immunodiagnosis of schistosomiasis by determination of the circulating antigens CAA and CCA, in particular in individuals with recent or light infections. Acta Trop 77:69-80

Van Remoortere A, Hokke CH, van Dam GJ, van Die I, Deelder AM, van den Eijnden DH (2000) Various stages of Schistosoma express Lewis ${ }^{\mathrm{x}}$, LacdiNAc, GalNAc $\beta 1-4($ Fuc $\alpha 1-3)$ GlcNAc and GalNAc $\beta 1-4$ (Fuc $\alpha 1-2$ Fuc $\alpha 1-3)$ GlcNAc carbohydrate epitopes: detection with monoclonal antibodies that are characterized by enzymatically synthesised neoglycoproteins. Glycobiology 86:410-413

Van Remoortere A, van Dam GJ, Hokke CH, van den Eijnden DH, van Die I, Deelder AM (2001a) Profiles of immunoglobulin $\mathrm{M}(\operatorname{IgM})$ and $\operatorname{IgG}$ antibodies against defined carbohydrate epitopes in sera of Schistosoma-infected individuals determined by surface plasmon resonance. Infect Immun 69:2396-2401

Van Remoortere A, Vermeer HJ, Agoston K, Kerékgyártó J, van Roon AM, Wilson RA, Langermans JAM, van Die I, van den Eijnden DH, Vliegenthart JFG, Kamerling JP, Hokke CH, van Dam GJ, Deelder AM (2001b) Analysis by surface plasmon resonance of anti-carbohydrate responses to synthetic glycan epitopes in Pan troglodytes vaccinated with irradiated Schistosoma mansoni cercariae. Glycoconj J 18: Abstract C16.11

Vermeer HJ, Halkes KM, Kamerling JP, Vliegenthart JFG (2000) Synthesis and conjugation of oligosaccharide fragments related to the immunologically reactive part of the circulating anodic antigen of the parasite Schistosoma mansoni. J Chem Soc, Perkin Trans 1: 2249-2263. 